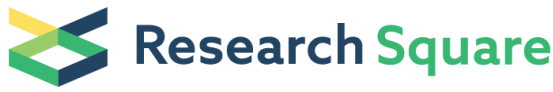 \\ Preprints are preliminary reports that have not undergone peer review. \\ They should not be considered conclusive, used to inform clinical practice, or referenced by the media as validated information.
}

\section{Decrease of exercise endurance in critically ill COVID-19 survivors: 4 case reports}

\section{Qian Geng}

Department of Pulmonary Diseases, State Key Laboratory of Respiratory Disease, National Clinical Research Center for Respiratory Disease, Guangzhou Institute of Respiratory Health, First Affiliated Hospital of Guangzhou Medical University

\section{Xinxin Yu}

Department of Pulmonary Diseases, State Key Laboratory of Respiratory Disease, National Clinical Research Center for Respiratory Disease, Guangzhou Institute of Respiratory Health, First Affiliated Hospital of Guangzhou Medical University

\section{Beilan Shen}

Department of Pulmonary Diseases, State Key Laboratory of Respiratory Disease, National Clinical Research Center for Respiratory Disease, Guangzhou Institute of Respiratory Health, First Affiliated Hospital of Guangzhou Medical University

\section{Shiyue Li}

Department of Pulmonary Diseases, State Key Laboratory of Respiratory Disease, National Clinical Research Center for Respiratory Disease, Guangzhou Institute of Respiratory Health, First Affiliated Hospital of Guangzhou Medical University

\section{Jinping Zheng}

Department of Pulmonary Diseases, State Key Laboratory of Respiratory Disease, National Clinical Research Center for Respiratory Disease, Guangzhou Institute of Respiratory Health, First Affiliated Hospital of Guangzhou Medical University

\section{Liping Zhong}

Department of Pulmonary Diseases, State Key Laboratory of Respiratory Disease, National Clinical Research Center for Respiratory Disease, Guangzhou Institute of Respiratory Health, First Affiliated Hospital of Guangzhou Medical University

\section{Yi Hong}

Department of Pulmonary Diseases, State Key Laboratory of Respiratory Disease, National Clinical Research Center for Respiratory Disease, Guangzhou Institute of Respiratory Health, First Affiliated Hospital of Guangzhou Medical University

\section{Xiaoyan Huang}

Department of Radiology, The First Affiliated Hospital of Guangzhou Medical University

\section{Qingsi Zeng}

Department of Radiology, The First Affiliated Hospital of Guangzhou Medical University

\section{Shaoqiang Li}


Department of Pulmonary Diseases, State Key Laboratory of Respiratory Disease, National Clinical Research Center for Respiratory Disease, Guangzhou Institute of Respiratory Health, First Affiliated Hospital of Guangzhou Medical University

\section{Feng Ye}

Department of Pulmonary Diseases, State Key Laboratory of Respiratory Disease, National Clinical Research Center for Respiratory Disease, Guangzhou Institute of Respiratory Health, First Affiliated Hospital of Guangzhou Medical University

\section{Weijie Guan}

Department of Pulmonary Diseases, State Key Laboratory of Respiratory Disease, National Clinical Research Center for Respiratory Disease, Guangzhou Institute of Respiratory Health, First Affiliated Hospital of Guangzhou Medical University

\section{Yanqing Xie}

Department of Pulmonary Diseases, State Key Laboratory of Respiratory Disease, National Clinical Research Center for Respiratory Disease, Guangzhou Institute of Respiratory Health, First Affiliated Hospital of Guangzhou Medical University

\section{Nanshan Zhong}

Department of Pulmonary Diseases, State Key Laboratory of Respiratory Disease, National Clinical Research Center for Respiratory Disease, Guangzhou Institute of Respiratory Health, First Affiliated Hospital of Guangzhou Medical University

\section{Yi Gao (D misstall2@163.com )}

Department of Pulmonary Diseases, State Key Laboratory of Respiratory Disease, National Clinical Research Center for Respiratory Disease, Guangzhou Institute of Respiratory Health, First Affiliated Hospital of Guangzhou Medical University

\section{Case Report}

Keywords: coronavirus disease 2019, Critically III, Pulmonary Function Test, Cardiopulmonary Exercise Test, exercise endurance

Posted Date: September 8th, 2020

DOI: https://doi.org/10.21203/rs.3.rs-72429/v1

License: (1) This work is licensed under a Creative Commons Attribution 4.0 International License. Read Full License 


\section{Abstract}

Background: The Coronavirus Disease 2019 (COVID-19) already have been as a pandemic. However, knowledge about the sequelae of severe acute respiratory syndrome coronavirus 2 (SARS-CoV-2) infection remains limited. Here we descirbe the pulmonary function test (PFT) and cardiopulmonary exercise test (CPET) of critically ill COVID-19 in four cases with sereve acute respiratory distress syndrome (ARDS) after discharge.

Case presentation: We introduce four patients who complained of fever, cough, chest tightness and other symptoms, all of them were confirmed as SARS-CoV-2 infection by real-time reverse transcription polymerase chain reaction (RT-PCR). They were treated with mechanical ventilation because of severe ARDS. After respiratory support, antiviral and anti-infective treatment, they were weaned from mechanic ventilation with the improvement of hypoxemia. All patients were discharged from the hospital after completion of treatment and had no mortality. Around 1-month post-discharge, they were followed up for chest computed tomography (CT) scan, and performed PFT and CPET. Peak oxygen uptake of predicted (peakVO $\mathrm{K}_{2} \%$ pred) decreased in all four cases, although spirometry were in the normal range, and only 2 cases had mild decline in carbon monoxide diffusion capacity of predicted (DLCO\%pred).

Conclusions: We found reduced exercise endurance in all four COVID-19 survivors, even parts of them with normal or slightly abnormal static lung function. We also believe that exercise endurance impairment of COVID-19 convalescents is more likely affected by extrapulmonary factors. Taken the above into consideration, our study highlights that the combination of PFT and CPET are important tests for tracking the development and recovery of COVID-19 survivors.

\section{Background}

Coronavirus disease 2019 (COVID-19) is a novel infectious disease caused by severe acute respiratory syndrome coronavirus 2 (SARS-CoV-2) ${ }^{[1]}$. It has been declared as a public health emergency of international concern. As of July 10, 2020, there were 12102328 confirmed cases had been documented worldwide ${ }^{[2]}$, the number of cases discharged is increasing continuously. For COVID-19 discharged patients, functional assessments such as exercise endurance and static lung function are highly recommended by the National Health Commission of China. At present, multiple studies on the epidemiology, virology, and clinical findings of COVID-19 had been reported globally ${ }^{[3]}$. However, very little literature regarding the functional injuries related to COVID-19 post-discharge, especially in critically ill patients. We describe the characteristics of cardiopulmonary exercise test (CPET) and pulmonary function tests (PFTs) including spirometry and diffusion capacity (QuarkPFT-CPET; COSMED, Italy) around 1-month post-discharge in four critically ill COVID-19 patients with severe acute respiratory distress syndrome (ARDS) admitted to our hospital from January to February 2020. All patients were confirmed with COVID-19 by presenting novel coronavirus. The research was approved by the ethics committee of the First Affiliated Hospital of Guangzhou Medical University, and all participants signed informed consent. 


\section{Case Presentations}

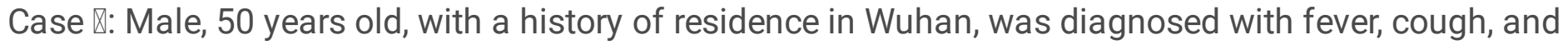
sputum on January 28, 2020. One day later, He complained that obvious shortness of breath, blood gas analysis showed that the oxygenation index $\left(\mathrm{OI}: \mathrm{PaO}_{2} / \mathrm{FiO}_{2}\right)$ seriously decreased to $84 \mathrm{mmHg}$, a chest computed tomography (CT) scan showed diffuse ground-glass opacities (GGOs) with interstitial septal thickening in bilateral lungs, all of which suggested that the patient had severe ARDS. So he received endotracheal intubation and mechanical ventilation quickly. On illness day 4 , he was transferred to the Intensive Care Unit (ICU) in our hospital, with an APACHE II score was 25. On illness day 20, the OI $(321 \mathrm{mmHg})$ of the patient improved, and he was weaned from mechanic ventilation successfully. He discharged on March 17. At his follow-up visit, 1-month after discharge from the hospital, he reported without any respiratory symptoms. Chest CT scan revealed bilateral GGOs which was better than before (Figure 1). There are neither restrictive nor obstructive of his spirometry. The diffusion capacity was slightly reduced. The patient completed the CPET. The maximal effort was evidenced by achieving a peak heart rate (HR) of $88 \%$, and respiratory exchange ratio (RER) of 1.34 , and clinically looking truly exhausted at peak exercise. Exercise stopped due to leg fatigue. The anaerobic threshold (AT) was normal using either the V-slope method (Figure. 2. c) or the ventilatory equivalents method (Figure. 2. d, g). There was plenty of breath reserve at peak exercise, the ventilatory equivalents for carbon dioxide at anaerobic threshold (VE/ $\left./ \mathrm{VCO}_{2} @ A T\right)$ and the slope of ventilatory equivalent for carbon dioxide $(\mathrm{VE} / \mathrm{VCO} 2$ slope) were normal, defining no ventilatory limitation to exercise. Meanwhile, there was no abnormal blood pressure response and no obvious ST-segment change in electrocardiogram (ECG). A peak oxygen uptake (peak $\mathrm{VO}_{2}$ ) of $58 \%$ predicted on a cycle ergometer noted a reduction in aerobic capacity. The results of PFTs and peak exercise are presented in Table 1, and the process diagram of CPET is shown in Figure. 2.

Case $\Downarrow:$ This was a 58 years old man who presented in the hospital because of fever, shortness of breath on 26 January 2020 . On illness day 6, with deteriorating respiratory failure, worsening $\mathrm{Ol}(68 \mathrm{mmHg})$, and progressing abnormalities on chest CT scan with bilateral patchy shadowing, he required intubation and mechanical ventilation. On illness day 8, he was transferred to ICU in our hospital and had an APACHE II score of 22. The patient was extubated with improved $\mathrm{Ol}(301 \mathrm{mmHg})$ on illness day 20 and was discharged from hospital 33 days later. Return to the hospital for follow-up 1-month post-discharge, the patient complained of a little cough and chest tightness after activity. A chest CT scan showed slightly absorption of multiple patchy opacities and GGOs. The values of the spirometric were normal. The diffusion function was slightly reduced. The CPET results showed no ischemic and arrhythmic changes in ECG, no abnormalities in ventilation function, and no reduction in gas exchange efficiency during exercise. But peakVO $\mathrm{O}_{2}$ and Oxygen pulse were reduced.

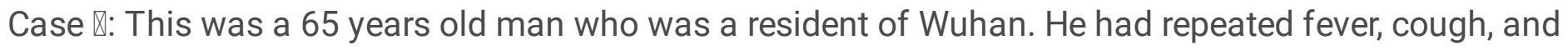
shortness of breath then was diagnosed on 22 January 2020. On illness day 2, he complained that dyspnea was worse than before, the blood gas analysis showing the OI was $77 \mathrm{mmHg}$, which was progressively aggravated. A Chest CT scan revealed bilateral infiltrates. So the patient received 
endotracheal intubation and mechanical ventilation to maintain respiratory motion. One day later, he was transferred to the ICU of our hospital with an APACHE II score of 16 and started treatment with antiinfection, anti-virus, and other supportive therapies. On illness day 19, the patient's OI (342mmHg) and chest CT significantly improved, so endotracheal intubation was removed. On illness day 49 , the patient recovered and discharged. He was followed up about 1-month post-discharge, and he complained of no cough, chest tightness, shortness of breath, dyspnea, and other discomforts. Bilateral GGOs could be observed in a chest CT scan. His PFTs were in the normal range. Exercise stopped because of leg fatigue, his AT, ventilation, gas exchange efficiency, and ECG had no abnormal changes during the exercise, but the endurance exercise capacity had decreased.

Case $\llbracket:$ male, 41 years old, was diagnosed due to fever and cough on January 28,2020 . The patient had a residence history in Wuhan. Given high fever and shortness of breath occurred again, a chest CT scan suggested bilateral pneumonia, so the patient was given intensified anti-infection treatment on illness day 9. However, the dyspnea of the patient continued to progress, the OI $(67 \mathrm{mmHg})$ decreased, and CT indicated the progression of the disease, so the patient was assisted by endotracheal intubation and transferred to ICU in our hospital on illness day 19. His highest APACHE II score while in the hospital was 16. He was prescribed antiviral and anti-infection treatment. The 32 days of illness his endotracheal intubation was removed successfully due to improved OI $(418 \mathrm{mmHg})$ and CT, then the patient was discharged on illness day 44. The patient showed no obvious symptoms of a 1-month follow-up after discharge. A chest CT scan showed a few patch shadows in the right upper lung. PFTs were within normal limits. During the CPET, the ECG showed no arrhythmia and ST-changes, AT, ventilation function and efficiency were normal, the patient's exercise endurance had decreased.

4 patients' clinical characteristics, PFTs, and CPET are shown in Table 1.

\section{Discussion And Conclusion}

An epidemiological analysis of 72,314 COVID-19 cases in China showed a large number of critically ill patients, although the proportion is relatively low $(5 \%)^{[5]}$. Given limited understanding of the pulmonary function and exercise endurance status in convalescents with COVID-19, we follow-up 4 critically ill patients with severe ARDS at 1-month post-discharge, and performed CT, spirometry, diffusion capacity and CPET for all patients. Noteworthy, despite the chest CT was significantly improved, the spirometry was normal, and carbon monoxide diffusion capacity (DLCO) was normal or only slightly abnormal in COVID-19 convalescents, the exercise endurance was still limited.

The pathological characteristics of COVID-19 had been shown bilateral diffuse alveolar damage with cellular fibromyxoid exudates, hyaline membrane formation, and intra-alveolar inflammatory exudates, partial alveolar exudate organization and pulmonary interstitial fibrosis formation ${ }^{[6]}$, which are similar to those seen in severe acute respiratory syndrome (SARS) and Middle Eastern respiratory syndrome (MERS) coronavirus infection, and the lung is the main organ primarily affected ${ }^{[6][7]}$. The impairment of diffusion capacity is associated with pathophysiological changes. Due to alveolar epithelial cell damage 
and membrane thickening, the diffusion area reduced, diffusion distance increased, and the diffusion capacity decreased accordingly.

Mo et al. ${ }^{[4]}$ reported impairment of diffusion capacity and restrictive ventilatory defect in non-critical COVID-19 patients at the time of hospital discharge. Anomalies were accounted for $9.1 \%$ in forced vital capacity of predicted (FVC \%pred) and accounted for $13.6 \%$ in forced expiratory volume in one second of predicted (FEV ${ }_{1} \%$ pred); The decrease was noted in DLCO \% predicted totally in $47.2 \%$ of the cases, with a significant difference in impaired diffusing capacity among the different groups of severity, which accounted for $30.4 \%$ in mild illness, $42.4 \%$ in pneumonia and $84.2 \%$ in severe pneumonia, respectively. However, in comparison to this study of non-critical cases at the time of discharge, our study of critically ill cases at 1-month post-discharge showed no abnormalities in spirometry in all patients and only mild diffusing impairment in 2 patients. It suggests that impaired lung function of COVID-19 patients might return over time.

All patients completed the CPET, in which the primary termination symptoms were leg fatigue in 3 patients and dyspnea in 1 patient. Interestingly, $\mathrm{PeakVO}_{2}, \mathrm{VO}_{2} / \mathrm{HR}$, and OUES decreased, while $\mathrm{BR}$ and $\mathrm{VE} / \mathrm{VCO}_{2}$ slope were normal, and no significant decrease was detected in $\mathrm{SpO}_{2}$ in all patients during exercise. It is indicated that the exercise endurance and aerobic metabolism capacity of the whole patients were reduced, nevertheless, nonventilatory limitation and no gas exchange inefficiency were noted in the exercise phase. The impairment of exercise capacity and static pulmonary function was inconsistent, besides the dynamic respiratory functions had no limitation, suggested the possibility that pulmonary dysfunction was not primarily a reason for the reduced exercise capacity. Therefore, we considered that exercise endurance impairment of COVID-19 convalescents is more likely affected by extrapulmonary factors, such as muscle weakness after long-term bed rest during hospitalization.

We reviewed previous studies of SARS, Ong et al. ${ }^{[8]}$ showed that $15 \%$ of the patients had restrictive pulmonary ventilation dysfunction, $39 \%$ of them had impairment diffusion function and $41 \%$ had decreased exercise endurance at 3 months after discharge. The result of exercise capacity also cannot be explained by impairment of pulmonary function. Our finding seems to be consistent with this study.

Our study has several limitations. First, the number of critical illness cases enrolled in our study was insufficient. Moreover, the PFT and CPET data were lack at the time of hospitalization and discharge. Finally, our study was only a short-term follow-up. Further investigations and long-term follow-up of more critically ill COVID-19 cases are urgently needed to reveal the clinical outcome and prognosis.

To our knowledge, this is the first report of the combination of PFT and CPET after COVID-19 rehabilitation. Our study indicates that exercise endurance may decrease in critically ill COVID-19 patients, even with normal or mild impairment in PFT and significant improvement of CT. It is not only PFT but also CPET should be considered in long-term follow-up, particularly for severe and critical illness COVID19 survivors, to more fully understand the impact of COVID-19 and provide functional assessment for rehabilitation. 


\section{Abbreviations}

ARDS: severe acute respiratory distress syndrome; AT: anaerobic threshold; BR: breath reserve; COVID-19: Coronavirus Disease 2019; CPET: cardiopulmonary exercise test; CT: chest computed tomography; DLCO\%pred: carbon monoxide diffusion capacity of predicted; $\mathrm{FEV}_{1} \%$ pred: forced expiratory volume in one second of predicted; FVC\%pred: forced vital capacity of predicted; OI: oxygenation index; ICU: Intensive Care Unit; OUES: Oxygen uptake efficiency slope; $\mathrm{PeakVO}_{2}$ of predicted: peak oxygen uptake of predicted; PFT: pulmonary function test; SARS-CoV-2: syndrome coronavirus 2; VE/VCO $\mathrm{VCAT}_{2}$ : Ventilatory equivalents for carbon dioxide at anaerobic threshold; $\mathrm{VE} / \mathrm{VCO}_{2}$ slope: the slope of ventilatory equivalent for carbon dioxide; $\mathrm{VO}_{2} / \mathrm{HR}$ of predicted: Oxygen pulse of predicted;

\section{Declarations}

Acknowledgements: None.

Authors' Contributions:YG, JPZ had full access to all of the data in the study and take responsibility for the accuracy of the data. QG, XXY, BLS, SYL and JPZ contributed equally to this manuscript. QG and YG wrote the manuscript. YG, JPZ, WJG and SYL reviewed and revised the manuscript. QG, XXY, LPZ, BLS and YQX contributed substantially to the study design, XXY and YH conducted CPET measurement. XYH and QSZ helped CT scanning and analysis. All authors read and approved the final manuscript.

Funding: This study was supported by Guangdong science and technology emergency project for the severe acute respiratory syndrome coronavirus 2 (SARS-CoV-2) infection prevention and control (2020B1111340014) and National Key R\&D plan (2018YFC1311900).

Ethics approval and consent to participate: The research was approved by the ethics committee of the First Affiliated Hospital of Guangzhou Medical University.

Consent to publish: Written informed consent was obtained from the patients for publication of this Case report and any accompanying images. A copy of the written consent is available for review by the Editor of this journal.

Availability of data and materials: All data generated or analysed during this study are included in this published article.

Competing interests: The authors declare that they have no competing interests.

\section{References}

[1] Zhu N, Zhang D, Wang W, Li X, Yang B, Song J, et al. A Novel Coronavirus from Patients with Pneumonia in China, 2019. N Engl J Med. 2020;382(8):727-733. 
[2] World Health Organization. Coronavirus disease (COVID-19) Situation Report-172.

https://www.who.int/docs/default-source/coronaviruse/situation-reports/20200710-covid-19-sitrep172.pdf?sfvrsn=70724b90_2. Accessed July 10, 2020.

[3] Guan WJ, Ni ZY, Hu Y, Liang WH, Ou CQ, He JX, et al. Clinical Characteristics of Coronavirus Disease 2019 in China. N Engl J Med. 2020;382(18):1708-1720.

[4] Mo X, Jian W, Su Z, Chen M, Peng H, Peng P, et al. Abnormal pulmonary function in COVID-19 patients at time of hospital discharge. Eur Respir J. 2020;55(6):2001217.

[5] Wu Z, McGoogan JM. Characteristics of and Important Lessons From the Coronavirus Disease 2019 (COVID-19) Outbreak in China: Summary of a Report of 72314 Cases From the Chinese Center for Disease Control and Prevention [published online ahead of print, 2020 Feb 24]. JAMA. https://doi.org/10.1001/jama.2020.2648.

[6] Zhang H, Zhou P, Wei Y, Yue H, Wang Y, Hu M, et al. Histopathologic Changes and SARS-CoV-2 Immunostaining in the Lung of a Patient With COVID-19. Ann Intern Med. 2020;172(9):629-632.

[7] Xu Z, Shi L, Wang Y, Zhang J, Huang L, Zhang C, et al. Pathological findings of COVID-19 associated with acute respiratory distress syndrome. Lancet Respir Med. 2020;8(4):420-422.

[8] Ong KC, Ng AW, Lee LS, Kaw G, Kwek SK, Leow MK, et al. Pulmonary function and exercise capacity in survivors of severe acute respiratory syndrome. Eur Respir J. 2004;24(3):436-42.

\section{Tables}

Table1: Clinical Characteristics, pulmonary function tests and cardiopulmonary exercise test at 1-month post-discharge follow-up 


\begin{tabular}{|c|c|c|c|c|}
\hline & Case 1 & Case & Case 1 & Case 1 \\
\hline Age & 50 & 58 & 65 & 41 \\
\hline Gender & male & male & male & male \\
\hline BMI & 25.4 & 23.4 & 21.6 & 28.2 \\
\hline & 10 & & & Never smoked \\
\hline $\begin{array}{l}\text { Comorbidity } \\
\text { Days in ICU }\end{array}$ & $\begin{array}{l}\text { Hypertension Diabetes } \\
40\end{array}$ & no & & $\begin{array}{l}\text { Hypertension } \\
24\end{array}$ \\
\hline Hospitalization days & 50 & 53 & 49 & $\begin{array}{l}24 \\
44\end{array}$ \\
\hline APACHEII scores & 25 & 22 & 16 & 16 \\
\hline $\begin{array}{l}\text { mMRC at follow-up } \\
\text { PFTs }\end{array}$ & 1 & 1 & 0 & 0 \\
\hline $\mathrm{FEV}_{1} \%$ pred & 92 & 99 & 115 & 87 \\
\hline $\begin{array}{l}\text { FVC\%pred } \\
\text { FEV }_{1} / \text { FVC }\end{array}$ & $\begin{array}{l}85 \\
87.3\end{array}$ & $\begin{array}{l}90 \\
87.7\end{array}$ & $\begin{array}{l}106 \\
85\end{array}$ & $\begin{array}{l}87 \\
83.8\end{array}$ \\
\hline $\mathrm{FEF}_{25 \%-75 \%} \%$ pred & 116 & 127 & 109 & 81 \\
\hline $\mathrm{FEF}_{50 \%} \%$ pred & 118 & 136 & 100 & 89 \\
\hline $\mathrm{FEF}_{75 \%} \%$ pred & 96 & 108 & 111 & 69 \\
\hline DLCO\%pred & 78 & 69 & 85 & 80 \\
\hline $\begin{array}{l}\text { KCO \%pred } \\
\text { CPET }\end{array}$ & 86 & 81 & 90 & 88 \\
\hline PeakVO $_{2} \%$ pred & 58 & 44 & 66 & 73 \\
\hline $\mathrm{VO}_{2} @ \mathrm{AT} \%$ pred & 42.8 & 43.5 & 52.2 & 50 \\
\hline OUES & 1509 & 1252 & 1465 & 2353 \\
\hline OUESロLLN" & $\begin{array}{l}2596 \\
537\end{array}$ & $\begin{array}{l}2201 \\
52\end{array}$ & 1905 & $\begin{array}{l}2707 \\
583\end{array}$ \\
\hline $\mathrm{VO}_{2} / \mathrm{HR} \%$ pred & 66 & & & \\
\hline $\mathrm{VE} / \mathrm{VCO}_{2} @ \mathrm{AT}$ & 31.3 & 34.6 & 30.4 & 28.7 \\
\hline $\mathrm{VE} / \mathrm{VCO}_{2}$ slope & 27.6 & 30.3 & 25.4 & 26 \\
\hline
\end{tabular}

APACHEII: acute physiology and chronic health evaluation II; PFTs: pulmonary function tests; $\mathrm{FEV}_{1}$ \%pred: forced expiratory volume in one second of predicted; FVC\%pred: forced vital capacity of predicted; $\mathrm{FEF}_{25 \%-75 \%} \%$ pred: mean forced expiratory flow between $25 \%$ and $75 \%$ of predicted; $\mathrm{FEF}_{50 \%} \%$ pred: Instantaneous forced expiratory flow when $50 \%$ of the FVC has been expired of predicted; DLCO\%pred: carbon monoxide diffusion capacity of predicted; KCO\%pred: carbon monoxide transfer coefficient of predicted; CPET: cardiopulmonary exercise test; PeakVO$_{2}$ \%pred: peak oxygen uptake of predicted; $\mathrm{VO}_{2} @ A T \%$ pred: Oxygen uptake at anaerobic threshold of predicted; OUES: Oxygen uptake efficiency slope; OUES(LLN): OUES lower limit of normal value; BR: breath reserve; $\mathrm{VO}_{2} / \mathrm{HR} \%$ pred: Oxygen pulse of predicted; $\mathrm{VE} / \mathrm{VCO}_{2} @ \mathrm{AT}$ : Ventilatory equivalents for carbon dioxide at anaerobic threshold; $\mathrm{VE} / \mathrm{VCO}_{2}$ slope: the slope of ventilatory equivalent for carbon dioxide.

\section{Figures}




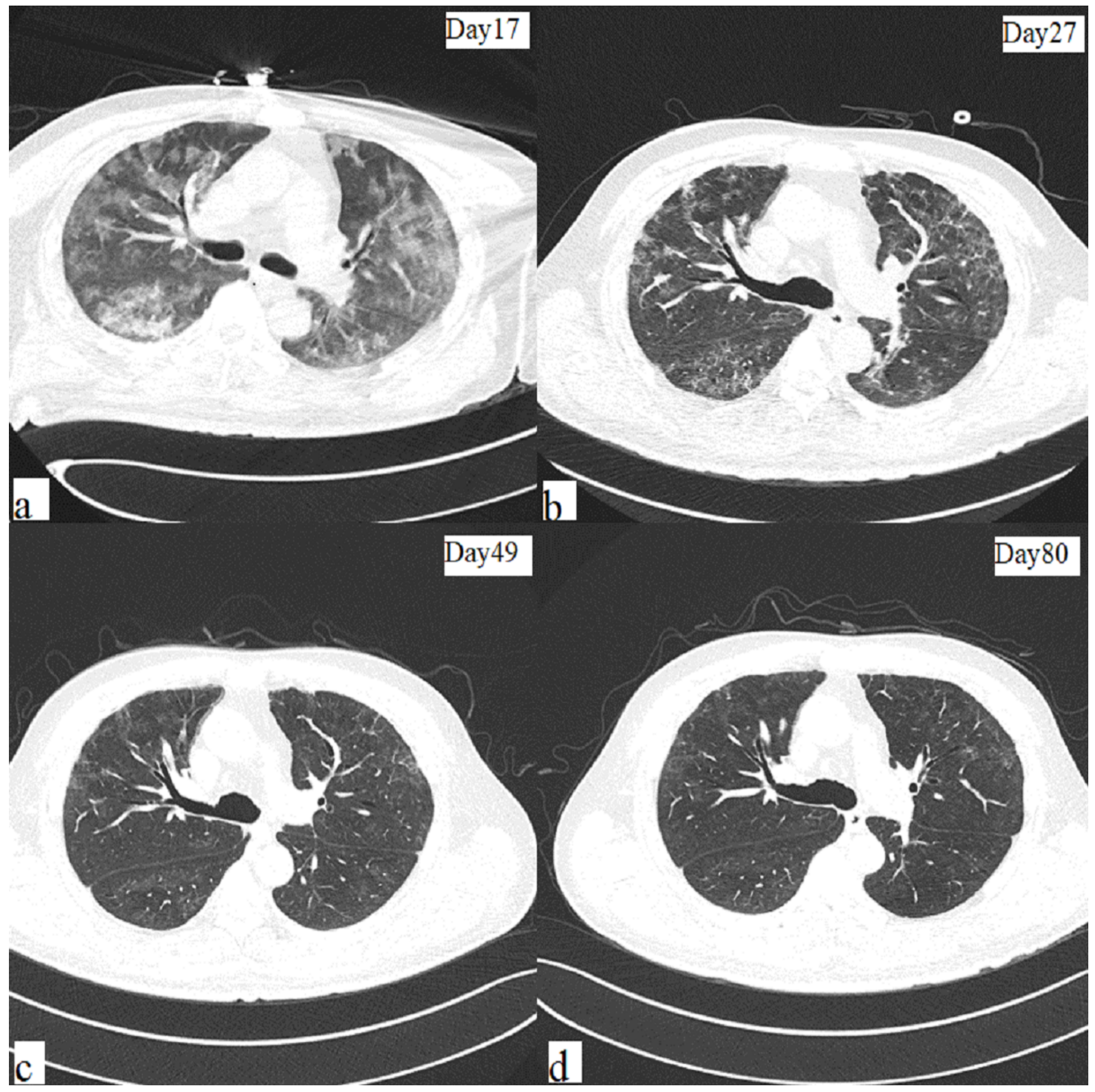

Figure 1

Transverse serial CT scans from case囚. a. Scan obtained on illness day 17 showed diffuse ground-glass opacities with interstitial septal thickening in bilateral lungs, mainly with the peripheral distribution. b. On illness day 27, the scan showed the absorption of abnormalities with diffuse ground-glass opacities left in both lungs and subpleural interstitial thickening. c. the day pre-discharge (day 49), the ground glass shadow and grid image were significantly less than before. d. 1-month after discharge (day 80), the grid shadow of both lungs decreased further. 

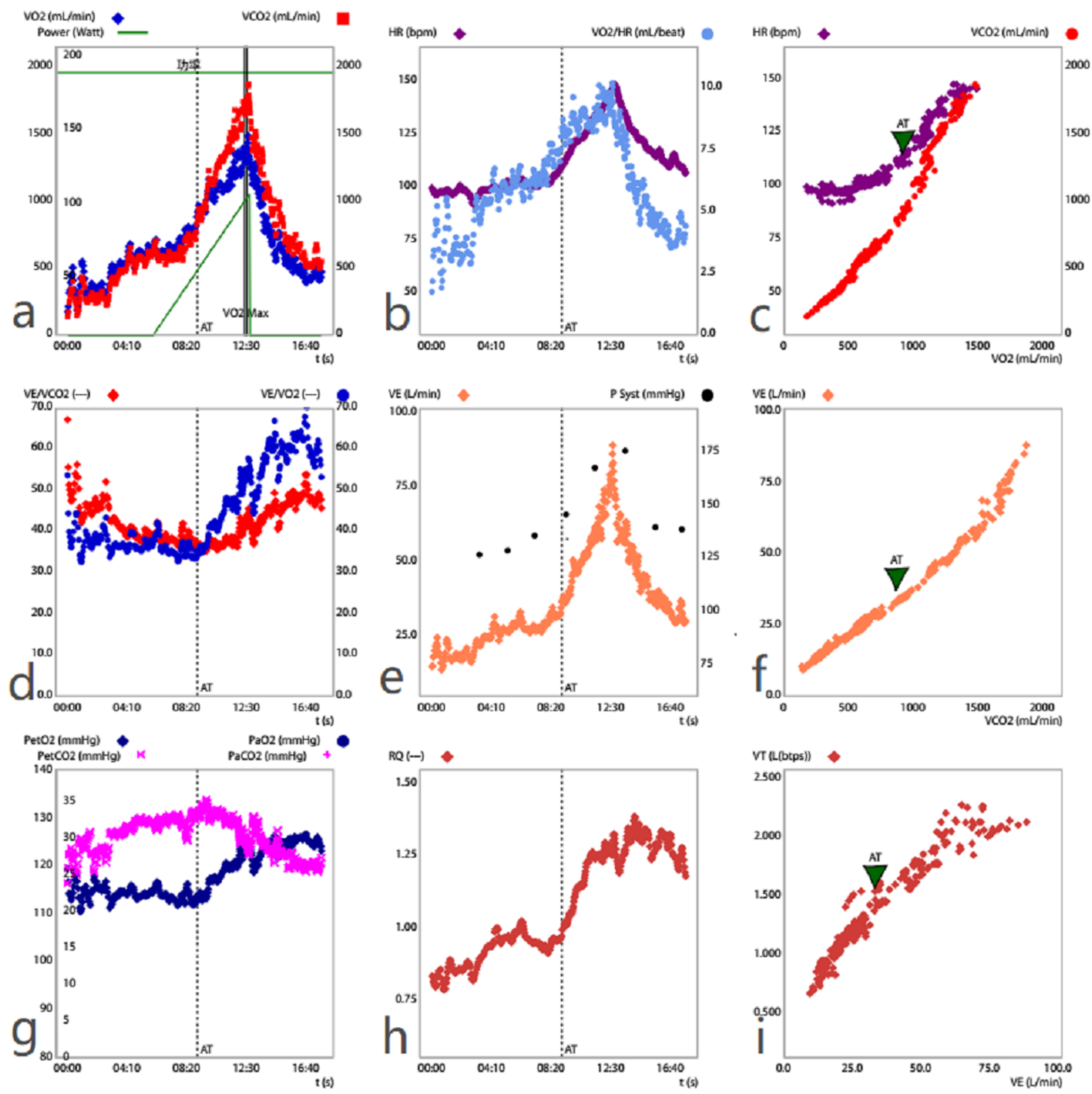

\section{Figure 2}

Graphic representation of the maximal, incremental, cardiopulmonary exercise response of case】. Exercise protocol: 15W/min. a. Oxygen uptake (VO2), carbon dioxide output (VCO2) and work rate (WR) are plotted over time. b. Heart rate (HR) and oxygen pulse ((VO2/HR) are plotted over time. c. HR and VCO2 are plotted over V02. $d$. The ventilatory equivalent for 02 (VE/VO2) and the ventilatory equivalent for CO2 (VE/VCO2) are plotted over time. e. VE and systolic blood pressure (SBP) are plotted as a function of time. f. VCO2 is plotted as a function of VE. g. End-tidal partial pressures of O2 (PET O2) and 
CO2 (PET CO2) are plotted over time. h. The respiratory exchange ratio (RER) plotted as a function of time. i. Tidal volume (VT) plotted as a function of VE. The AT point was determined by the V-slope method (Figure.2.c) or the ventilation equivalent method (Figure.2.d,g). 